\title{
Other Iatrogenic Immunodeficiency-Associated Lymphoproliferative Disorders with a T- or NK-cell phenotype
}

\author{
Akira Satou, ${ }^{1)}$ Toyonori Tsuzuki ${ }^{11}$ and Shigeo Nakamura ${ }^{2)}$
}

Other iatrogenic immunodeficiency-associated lymphoproliferative disorders (OIIA-LPDs) with a T- or NK-cell phenotype are markedly rare, with only a limited number of cases having been reported thus far. Methotrexate (MTX) is the most common agent used for OIIA-LPD patients, and 43 cases of MTX-associated T-LPDs (MTX T-LPDs) and five cases of MTX-associated NK/T-LPDs (MTX NK-LPDs) have been described. In addition to MTX T-LPDs and MTX NK/T-LPDs, T-LPD and NK/ T-LPDs have been reported in patients receiving other immunosuppressive agents such as thiopurines, TNF antagonists, and cyclosporine. Hepatosplenic T-cell lymphoma (HSTL) is specifically associated with iatrogenic immunodeficiency, and $10 \%$ of HSTL cases develop in patients receiving thiopurines and/or TNF antagonists for inflammatory bowel disease (IBD). In this review, we focused on MTX T-LPD, MTX NK/T-LPD, and HSTL in patients with IBD. These T- and NK/T-cell associated OIIA-LPDs are the most common in daily medical practice.

Keywords: other iatrogenic immunodeficiency-associated lymphoproliferative disorder, T or NK/T-cell lymphoma, methotrexate, inflammatory bowel disease, hepatosplenic T-cell lymphoma

\section{INTRODUCTION}

Immunodeficiency plays a key role in the pathogenesis of some lymphoproliferative disorders (LPDs). Immunodeficiency is caused by aging, primary immune disorders, HIV infection, and immunosuppressive drugs. ${ }^{1}$ In the 2017 WHO classification, ${ }^{1}$ LPDs that are associated with immunosuppressive agents are termed post-transplant LPDs (PTLDs) or other iatrogenic immunodeficiency-associated LPDs (OIIALPDs). OIIA-LPDs are defined as lymphoid proliferations or lymphomas that develop in patients receiving immunosuppressive drugs for an autoimmune disease or conditions other than post-transplantation. OIIA-LPDs are a heterogeneous group mainly consisting of polymorphic B-cell LPDs (B-LPDs), monomorphic LPDs, and Hodgkin lymphoma (HL). These OIIA-LPDs are often diagnostically and therapeutically challenging for both pathologists and clinicians. Monomorphic LPDs include cases that fulfill the criteria of diffuse large cell lymphoma, follicular lymphoma, peripheral T-cell lymphoma (PTCL), or extranodal natural killer (NK)/ T-cell lymphoma, nasal type. Cases accompanying Epstein-Barr virus (EBV)-positive $\left(\mathrm{EBV}^{+}\right)$mucocutaneous ulcers in patients receiving immunosuppressive drugs are also considered OIIA-LPD.

OIIA-LPDs are mainly B-LPD or HL types, whereas T-cell LPDs (T-LPDs) and NK/T-cell LPDs (NK/T-LPDs) are relatively rare. Methotrexate (MTX) is the most common agent used for OIIA-LPD patients. Previous large studies revealed that T-LPDs or NK/T-LPDs account for only $4-8 \%$ of MTX-associated LPDs. ${ }^{1-5}$ Currently, only 43 cases of MTX-associated T-LPDs (MTX T-LPDs) ${ }^{6-15}$ and five cases of

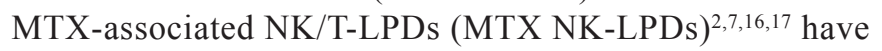
been described. In addition to MTX T-LPDs and MTX NK/ T-LPDs, T-LPD and NK/T-LPDs have been reported in patients receiving other immunosuppressive agents such as thiopurines, TNF antagonists, and cyclosporine. ${ }^{1,18}$ Hepatosplenic T-cell lymphoma (HSTL) is specifically associated with iatrogenic immunodeficiency, and 10\% of HSTL cases develop in patients receiving thiopurines and/or TNF antagonists for inflammatory bowel disease (IBD). ${ }^{19-21}$

In this review, we summarized the pathological and clinical aspects of OIIA-LPDs with a T-cell or NK/T-cell phenotype.

\footnotetext{
Received: March 25, 2019. Revised: April 20, 2019. Accepted: May 27, 2019. Online Published: June 28, 2019

DOI:10.3960/jslrt.19013

${ }^{1}$ Department of Surgical Pathology, Aichi Medical University Hospital, Nagakute, Japan, ${ }^{2}$ Department of Pathology and Laboratory Medicine, Nagoya University Hospital, Nagoya, Japan

Corresponding author: Akira Satou, Department of Surgical Pathology, Aichi Medical University Hospital, 1-1, Yazakokarimata, Nagakute, 480-1195, Japan.

E-mail: satoakira@aichi-med-u.ac.jp

Copyright (C) 2019 The Japanese Society for Lymphoreticular Tissue Research

(cc) BY-NC-SA This work is licensed under a Creative Commons Attribution-NonCommercial-ShareAlike 4.0 International License.
} 
We focused on MTX T-LPDs, MTX NK/T-LPDs, and HSTL in patients with IBD. These T- and NK/T-cell-associated OIIA-LPDs are the most common in daily medical practice.

\section{MTX-ASSOCIATED LPDS WITH A T- OR NK-CELL PHENOTYPE}

MTX is an anti-rheumatic drug that is administered to patients with autoimmune diseases, particularly rheumatoid arthritis (RA). MTX suppresses the hyper-immune state of RA patients and is an excellent inhibitor of articular destruction. Therefore, MTX is currently used as a first-line anchor drug for RA therapy. ${ }^{22}$ However, the immunosuppressive state induced by MTX leads to the development of LPDs, and is the cause of MTX-associated LPDs, although the mechanism for its development is unclear. In addition, patients with RA develop LPDs 2.0- to 5.5-times more often than the general population..$^{23-25}$ The hyper-immune state of RA may play a role in the tumorigenesis of LPDs. Therefore, how MTX influences the development of LPDs remains controversial. On the other hand, a significant proportion of patients with MTX-associated LPDs, particularly EBV ${ }^{+}$ patients, have presented spontaneous regression (SR) after MTX cessation. ${ }^{2-5,26}$ This phenomenon is characteristic of MTX-associated LPDs and is regarded as strong evidence for a potential tumorigenic role of MTX.

Since MTX-associated LPD was first documented in 1993, ${ }^{27}$ a number of cases have been reported. However, MTX-associated LPDs are mainly B-LPD or HL type, whereas T-LPDs are relatively rare (4-8\%). Indeed, only a limited number of T- LPD and NK/T-LPD cases have been reported in detail. ${ }^{6-17}$ The pathological and clinical aspects of MTX T-LPDs and MTX NK/T-LPDs are summarized below. In this report, we defined MTX-LPD as LPD that developed in patients receiving MTX at the time of diagnosis.

\section{MTX-associated T-LPD}

A total of 43 cases of MTX T-LPD have been reported to date. In general, the criteria for subclassifying MTX T-LPDs and NK/T-LPDs are the same as for T- and NK/T-LPDs in immunocompetent patients. ${ }^{1}$ The original diagnoses of these 43 cases were: 25 cases of angioimmunoblastic T-cell lymphoma (AITL), 6 PTCL, not otherwise specified (PTCLNOS), 4 EBV-CD8 ${ }^{+}$T-LPD, 2EBV ${ }^{+} \mathrm{CD}^{+}$T-LPD, and one each of anaplastic large cell lymphoma (ALCL), $\mathrm{EBV}^{+} \mathrm{CD} 30^{+}$ cutaneous T-cell lymphoma (CTCL), subcutaneous panniculitis-like T-cell lymphoma (SPTCL), EBV ${ }^{+}$SPTCL, $\mathrm{EBV}^{+} \mathrm{CD} 30^{+} \mathrm{T}-\mathrm{LPD}$, and adult T-cell leukemia/lymphoma (ATLL). Taking into account the histological and immunohistochemical findings, they were separated into the following types: AITL $(n=25)$, PTCL-NOS $(n=6), \mathrm{EBV}^{+} \mathrm{CD}^{+}$ T-LPD $(n=5), \operatorname{EBV}^{-} \mathrm{CD} 8^{+}$T-LPD $(n=5), \operatorname{ALCL}(n=1)$, and $\operatorname{ATLL}(n=1)$. The cases with $\mathrm{EBV}^{+} \mathrm{CD} 30^{+} \mathrm{CTCL}, \mathrm{EBV}^{+}$ SPTCL, and $\mathrm{EBV}^{+} \mathrm{CD} 30^{+} \mathrm{T}-\mathrm{LPD}$, which all expressed $\mathrm{CD} 8^{+}$, were included in $\mathrm{EBV}^{+} \mathrm{CD} 8^{+} \mathrm{T}-\mathrm{LPD}$; the case with SPTCL was included in $\mathrm{EBV}^{-} \mathrm{CD} 8^{+} \mathrm{T}-\mathrm{LPD}$.

\section{Histological and immunohistochemical findings in MTX T-LPD}

As stated above, the largest category of MTX T-LPDs includes cases that are classified as the AITL type (MTXAITL). The histological and immunohistochemical features of MTX-AITL are almost the same or similar to those of AITL in immunocompetent patients. MTX-AITL patients exhibited diffuse and polymorphous infiltration of small- to medium-sized lymphocytes intermingled with plasma cells, histiocytes, and eosinophils, accompanied by the proliferation of high endothelial venules (Figure 1A). Small- to medium-sized lymphocytes were characterized by clear cytoplasm (Figure 1B). Immunohistochemically, most cases were positive for $\mathrm{CD} 3, \mathrm{CD} 4$, the follicular helper T-cell markers, such as PD-1 (Figure 1C), and CXCL13, but negative for CD8.

The second largest category is the $\mathrm{CD} 8^{+} \mathrm{T}-\mathrm{LPD}$, including both $\mathrm{EBV}^{+}$and $\mathrm{EBV}^{-}$cases. Histologically, $\mathrm{CD}^{+}$ T-LPDs are mostly characterized by infiltration of medium-sized atypical lymphocytes (Figure 2A). Immunohistochemically, all cases were positive for CD3 and CD8 (Figure 2B). In addition, all of the cases examined were positive for the cytotoxic molecule (Figure 2C), but were negative for CD56 regardless of EBV positivity.

The PTCL-NOS type is a heterogeneous group that includes cases that are difficult to precisely categorize. Previously reported cases exhibited diffuse infiltration of medium- to large-sized atypical lymphocytes. The one case of ALCL was characterized by the proliferation of CD30 atypical large lymphocytes that were positive for CD3, TIA1 , and CD8, but negative for CD4. Only one case of ATLL type has been reported thus far. This case was morphologically characterized by the diffuse proliferation of mediumsized abnormal lymphocytes. The tumor cells were positive for CD3 and CD4, but negative for CD20, CD8, and CD30. Southern blotting for the HTLV-1 provirus revealed monoclonal proliferation of HTLV-1-infected cells.

\section{Clinical characteristics of MTX T-LPD}

The 43 MTX T-LPD patients consisted of 30 men and 13 women with a median age of 66 years (range, 31-85). Among them, 42 were treated for RA and one for polymyalgia rheumatica. Information of duration of MTX use was available for 33 patients, and the median time was 4 years (range, 0.5-21). For 6 of 16 patients with available data, iguratimoid, mizoribine, salazosulfapyridine, etanercept, and bucillamine were used as immunosuppressive agents in addition to MTX. At the time of diagnosis, 38 patients $(88 \%)$ had lymphadenopathy, including 12 who also had extranodal involvement. The remaining 5 patients $(12 \%)$ had only extranodal lesions as follows: skin $(n=2)$, subcutis $(n=1)$, subcutis and abdominal cavity $(n=1)$, and oral cavity $(n=1)$. T-cell and NK-cell PTLDs were reported to have a late onset after organ transplantation. Satou et al., however, recently reported that there was no significant difference in the MTXuse duration between MTX T- and B-LPD. ${ }^{6}$ 

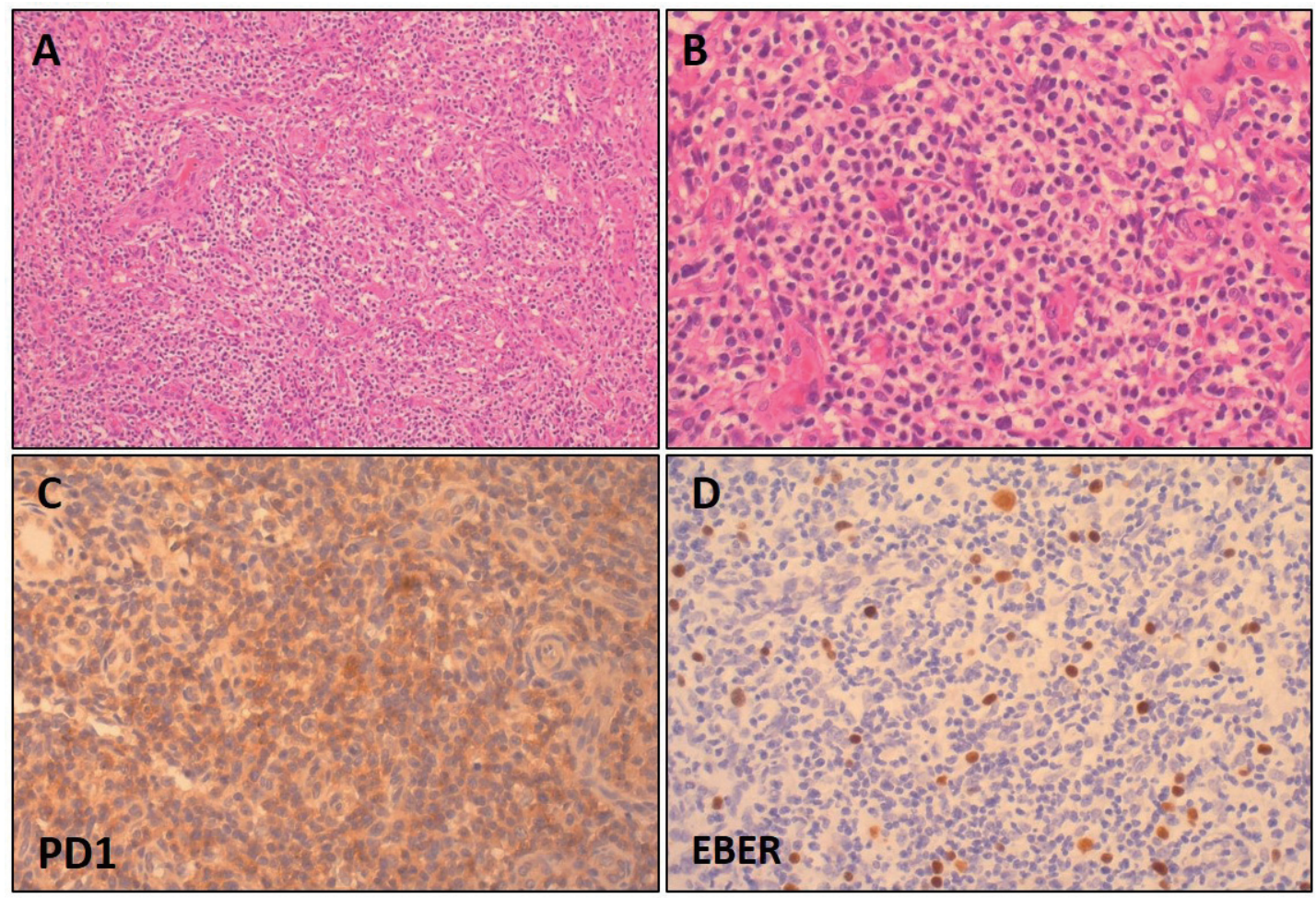

Fig. 1. Histological and immunohistochemical features of angioimmunoblastic T-cell lymphoma-type methotrexate-associated T-cell lymphoproliferative disorder (MTX-AITL).

MTX-AITL had diffuse and polymorphous infiltration of small- to medium-sized lymphocytes accompanied by the proliferation of high endothelial venules. Small- to medium-sized lymphocytes were characterized by clear cytoplasm ( $\boldsymbol{A}$ : HE x 200, B: HE x 400). Immunohistochemically, the tumor cells were positive for PD1 (C: PD1 x 200). Scattered EBV-infected B cells were detected in the background (D: EBER x 200).

\section{Therapy and prognosis for MTX T-LPDs}

After the diagnosis of MTX T-LPD, MTX was immediately withdrawn in 40 patients and $33(83 \%)$ presented with SR (complete remission [CR] or partial response [PR]) after cessation. Ten patients received cytotoxic chemotherapy as the initial treatment, including two patients who developed SR after MTX cessation. Eventually, 38 patients achieved $\mathrm{CR}$ and two achieved partial response PR. Nine of the 40 patients had relapse or progression. Notably, all $10 \mathrm{CD}^{+}$ T-LPD patients achieved CR after cessation of MTX regardless of EBV positivity. Furthermore, none of these 10 patients relapsed or required cytotoxic chemotherapy during their entire clinical course.

\section{EBV infection in MTX T-LPDs}

The EBV status was assessed in all of the previous cases. Five of 43 cases had $\mathrm{EBV}^{+}$tumor cells. Notably, all of the $\mathrm{EBV}^{+}$cases were exclusively $\mathrm{CD}^{+} \mathrm{T}-\mathrm{LPD}$ (Figure 2D). A recent paper revealed that patients with MTX T-LPDs had a significantly lower proportion of $\mathrm{EBV}^{+}$tumor cells than those with MTX B-LPDs. Among the $38 \mathrm{EBV}^{-}$cases in the present series, 32 (84\%) had scattered EBV-infected B cells in the background (Figure 1D). The reactivation of EBV in the background B cells is suggestive of the immunodeficient status of the patients.

\section{MTX-associated NK/T-LPDs}

Only five cases of MTX NK/T-LPD have been described thus far. ${ }^{2,7,16,17}$ The clinical features are summarized in Table 1. The five cases consisted of one male and four female patients with a median age of 73 years (range, 55-85). The primary sites were the nasal cavity $(n=2)$, nose $(n=1)$, gingiva $(\mathrm{n}=1)$, and both lungs $(\mathrm{n}=1)$. The histological and immunohistochemical features are summarized in Table 2. The features of MTX-associated NK-LPD were identical to those of extranodal NK/T-lymphoma. The size of lymphoma cells varied from medium to large. One case with a detailed description of histological features was accompanied by necrosis. Immunohistochemically, all of the cases with data were positive for CD3 and cytotoxic molecule (TIA-1 and/or granzyme B). Two of three cases were positive for CD8, one of three was positive for CD56, and all cases were $\mathrm{EBV}^{+}$. These $\mathrm{CD}^{+} \mathrm{MTX}$ NK/T-LPDs may overlap with $\mathrm{EBV}^{+} \mathrm{CD} 8^{+}$ MTX T-LPDs, and it was difficult to make a clear line between the two. For that reason, we followed the diagnoses given in the cited papers.

MTX was immediately withdrawn for all patients, and they all presented with SR after cessation except for one patient (case no.4) who received radiotherapy and achieved CR. Case no.5 first presented partial response (PR), but the tumor subsequently progressed. The patient received SMILE therapy (combination of steroid, methotrexate, ifosfamide, 


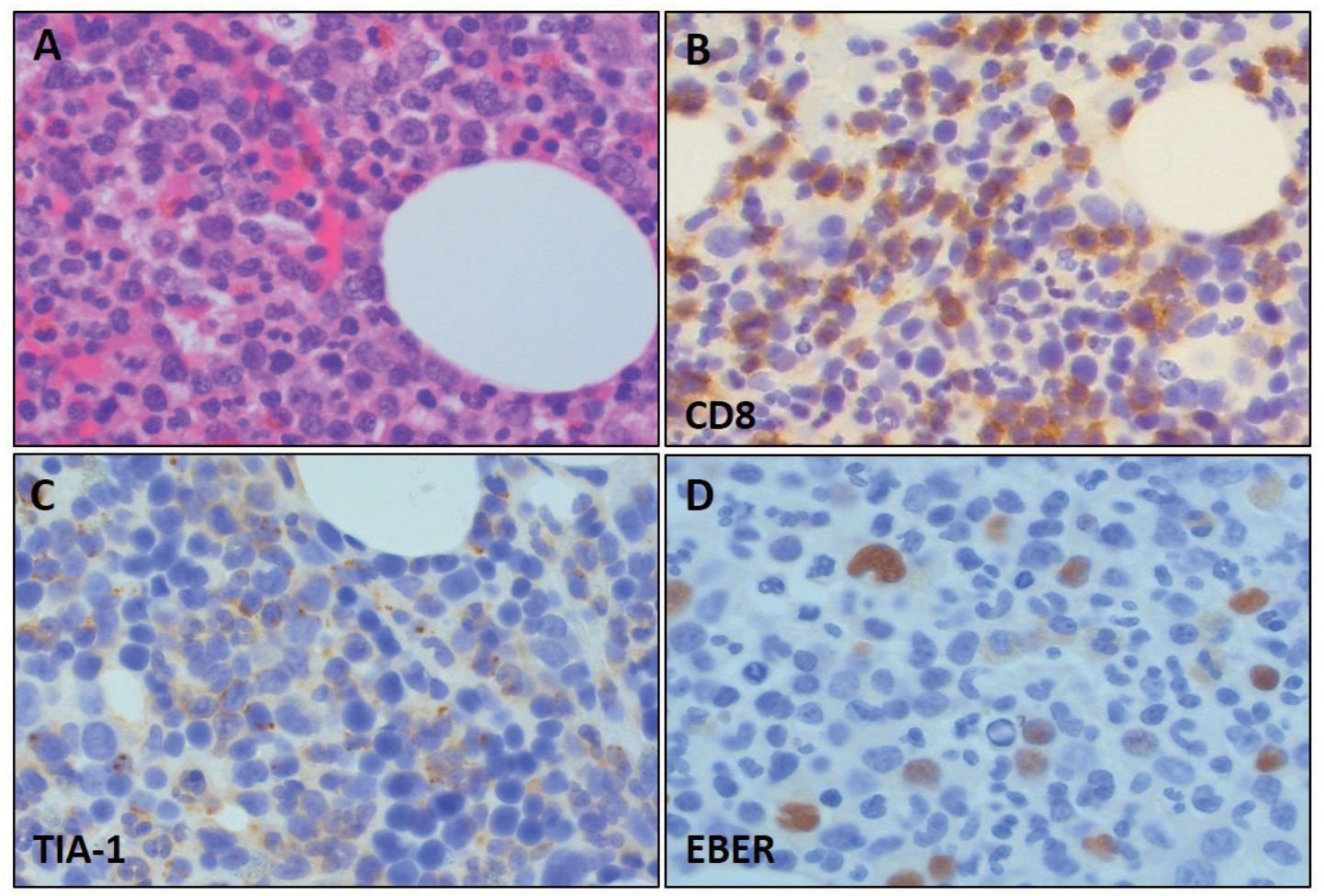

Fig. 2. Histological and immunohistochemical features of $\mathrm{EBV}^{+} \mathrm{CD} 8^{+}$cytotoxic T-cell lymphoma (CTL)-type methotrexate-associated T-cell lymphoproliferative disorder.

The $\mathrm{EBV}^{+} \mathrm{CD} 8^{+} \mathrm{CTL}$ type was characterized by the infiltration of medium-sized atypical lymphocytes $(A$ : HE x 400). Immunohistochemically, tumor cells were positive for CD8 (B: HE x 400), TIA-1 ( $\boldsymbol{C}$ : HE x 400), and $\operatorname{EBER}(\boldsymbol{D}$ : EBER x 400).

Table 1. Clinical features of methotrexate-associated NK/T-cell lymphoproliferative disorders

\begin{tabular}{|c|c|c|c|c|c|c|c|c|c|}
\hline Case & Age/sex & Primary site & $\begin{array}{l}\text { MTX duration } \\
\text { (years) }\end{array}$ & $\begin{array}{c}\text { First-line } \\
\text { Management }\end{array}$ & SR & Res & $\begin{array}{l}\text { Recurrence or } \\
\text { progression }\end{array}$ & $\begin{array}{c}\text { Additional } \\
\text { therapy }\end{array}$ & Outcome \\
\hline 1 & $74 / F$ & Nasal cavity & 9.2 & Off MTX & + & $\mathrm{CR}$ & No & No & Alive, $21 \mathrm{mo}$ \\
\hline 2 & $73 / F$ & Gingiva & NA & Off MTX & + & $\mathrm{CR}$ & No & No & Alive, $18 \mathrm{mo}$ \\
\hline 3 & $55 / \mathrm{F}$ & Nose & NA & Off MTX & + & $\mathrm{CR}$ & No & No & Alive, $36 \mathrm{mo}$ \\
\hline 4 & $85 / \mathrm{M}$ & Nasal cavity & 6 & $\begin{array}{c}\text { Off MTX } \\
\text { Radiotherapy }\end{array}$ & - & $\mathrm{CR}$ & NA & NA & NA \\
\hline 5 & $64 / \mathrm{F}$ & Bilateral lungs & 12 & Off MTX & + & PR & Yes & SMILE & Alive, under treatment $2 \mathrm{mo}$ \\
\hline
\end{tabular}

CR: complete response; F: female; M: male; mo: months; MTX; methotrexate; NA: not available; PR: partial response; SR: spontaneous regression.

Table 2. Histological and immunohistochemical features of MTX-associated NK/T-cell lymphoproliferative disorders

\begin{tabular}{ccccccccc}
\hline Case & Age $(\mathrm{y}) / \mathrm{sex}$ & Lymphoid size & CD3 & CD4 & CD8 & CM & CD56 & EBV \\
\hline 1 & $74 / \mathrm{F}$ & NA & NA & NA & NA & NA & NA & + \\
2 & $73 / \mathrm{F}$ & Pleo & + & + & + & + & - & + \\
3 & $55 / \mathrm{F}$ & Large & + & - & - & + & + \\
4 & $85 / \mathrm{M}$ & Med to Large & + & ND & ND & + & equivocal & + \\
5 & $64 / \mathrm{F}$ & Med & + & - & + & + & + & + \\
\hline
\end{tabular}

CM: cytotoxic molecule; EBV: Epstein-Barr virus; F: female; M: male; Med: medium; NA: not available; Pleo: pleomorphic 
1-asparaginase, and etoposide) as additional treatment. The disease was evaluated as PR after two courses of SMILE therapy, but there were no follow-up data.

\section{How MTX-associated T- and NK/T-LPDs should be treated}

As stated above, although some cases may relapse or progress later, SR may be expected in the majority of MTX T-LPD and NK/T-LPD cases. In particular, all of the CD8 ${ }^{+}$ T-LPD patients achieved CR after MTX cessation without relapse. It is well known that a significant proportion of patients with MTX B-LPD and HL types present SR after MTX cessation. ${ }^{2-5,26}$ Therefore, chemotherapy cannot be started immediately after the diagnosis of MTX B-LPD and HL type. In addition, withdrawal of MTX should be the initial management after the diagnosis of MTX T-LPDs and NK/T-LPDs, although a definite conclusion cannot be made yet. For patients that do not exhibit SR, or develop relapse or progression after SR, an aggressive therapy suitable for each lymphoma subtype is needed.

\section{Pathogenesis of MTX T- and NK/T- LPDs}

The genetic and molecular characteristics of MTX T- and NK/T-LPDs are unclear, and their pathogenesis remains to be elucidated. Possible mechanisms of their tumorigenesis are described below.

In general, regardless of cell lineage and EBV status, the immunosuppressive state induced by MTX is considered a common cause of MTX-associated LPDs. Feng et al. ${ }^{28}$ suggested another potential cause of $\mathrm{EBV}^{+} \mathrm{MTX}$-associated LPDs. They indicated that, in contrast to other causes of immunodeficiency, MTX may directly reactivate latent EBV, leading to the development of LPDs. However, most MTX T-LPDs are negative for EBV in proliferative T- and NK-cells, meaning that this model cannot directly be applied. The majority of EBV- MTX T-LPDs had scattered EBVinfected B cells in the background; therefore, the function of EBV-specific cytotoxic T lymphocytes may be suppressed due to immunodeficiency. ${ }^{29}$ Thus, the reactivation of EBV implies that the patients have an immune disorder and may suppress any immune response to inhibit tumor growth.

In cases of $\mathrm{EBV}^{-} \mathrm{CD}^{+} \mathrm{T}-\mathrm{LPD}$, the proliferation of $\mathrm{CD}{ }^{+}$ cytotoxic T-cell lymphoma cells may be induced by the growth of EBV-infected B cells. The CD8 ${ }^{+} \mathrm{T}$-cells act to prevent the transformation of EBV-infected B cells into a B-cell malignancy. ${ }^{30}$ In addition, Sandhu et al..$^{31}$ reported that MTX preferentially affects subsets of $\mathrm{CD} 8^{+} \mathrm{T}$ lymphocytes. They revealed that, after treatment with MTX, there was a significant decline in $\mathrm{CD} 8^{+} \mathrm{IFN} \gamma^{+}$and increase in CD $8^{+}$IL $17^{+}$T-cells. The ability of MTX to increase a subset of $\mathrm{CD}^{+} \mathrm{T}$-cells may also aid in the development of the $\mathrm{CD}^{+}$ T-LPD type. Future studies are expected to clarify these issues.

\section{HSTL IN PATIENTS WITH INFLAMMATORY BOWEL DISEASE}

Farcet et al. first described HSTL in 1990 as a PTCL of $\gamma \delta$ phenotype with tumor cells localized in the liver and spleen. ${ }^{32}$ Subsequently, HSTL with a $\alpha \beta$ phenotype was also reported. ${ }^{33}$ The majority of HSTL cases express $\gamma \delta$ T-cell receptors, and only a minority of cases are the $\alpha \beta$ type. HSTL is a rare and fatal extranodal lymphoma that primarily affects young men. It accounts for $<1 \%$ of all non-Hodgkin lymphomas and $1-2 \%$ of all PTCL cases. Patients typically present splenomegaly and hepatomegaly. Bone marrow involvement is detected in almost all patients. It is known that $20 \%$ of HSTL cases develop during chronic immune suppression, and $10 \%$ occur in individuals receiving thiopurines and/or TNF antagonists for IBD. ${ }^{1,19,34}$

Thiopurines (e.g. azathioprine and 6-mercaptopurine) and TNF antagonists (e.g. infliximab, adalimumab, and etanercept) are now widely used for IBD patients, including those with Crohn's disease (CD) and ulcerative colitis (UC). Herrinton et al. ${ }^{34}$ reported that IBD alone is not associated with a risk of lymphoma. However, previous reports suggested that the use of thiopurines and TNF antagonists, alone or in combination, was associated with an increased risk of lymphoma in IBD patients. ${ }^{34-38}$ According to the report of Herrinton et al., the majority of lymphomas developing in IBD patients were B-cell lymphomas and HL. The T-cell lymphomas in IBD patients were HSTL and mycosis fungoides, which accounted for $5 \%$ and $2 \%$ of the lymphomas arising in IBD, respectively. ${ }^{34}$ Considering that HSTL accounts for only $1-2 \%$ of all PTCLs, using these immunosuppressive agents may increase the risk for developing this rare lymphoma.

We identified 52 previously reported cases of HSTL in IBD patients. ${ }^{35,39-50}$ The pathological and clinical aspects of HSTL in IBD are summarized below.

\section{Clinicopathological findings of HSTL in IBD patients}

The 52 cases of HSTL were in 46 male and 6 female IBD patients with a median age of 23 years (range, 12-79). Among the 52 patients, 45 were treated for $C D$ and seven for UC. All of the patients received thiopurines and/or TNF antagonists, or other immunosuppressive agents. Eighteen patients received only thiopurines, one received only TNF antagonist, and 32 received both.

Histologically, all cases were consistent with the histopathological findings documented in HSTL. Namely, the tumor cells were small to medium in size with pale cytoplasm (Figure 3A). In the spleen, the neoplastic cells involved the cords and sinuses of expanded red pulp. The liver demonstrated predominant sinusoidal infiltration (Figure 3B). The bone marrow contained neoplastic cells in most cases, and exhibited an interstitial and sinusoidal pattern of involvement. Immunohistochemically, most cases were positive for CD3 (Figure 3C), CD8, and TIA-1 (Figure 3D). TCR expression was evaluated in 24 cases: $\gamma \delta$ phenotype in 18 and $\alpha \beta$ phenotype in 6 cases. 


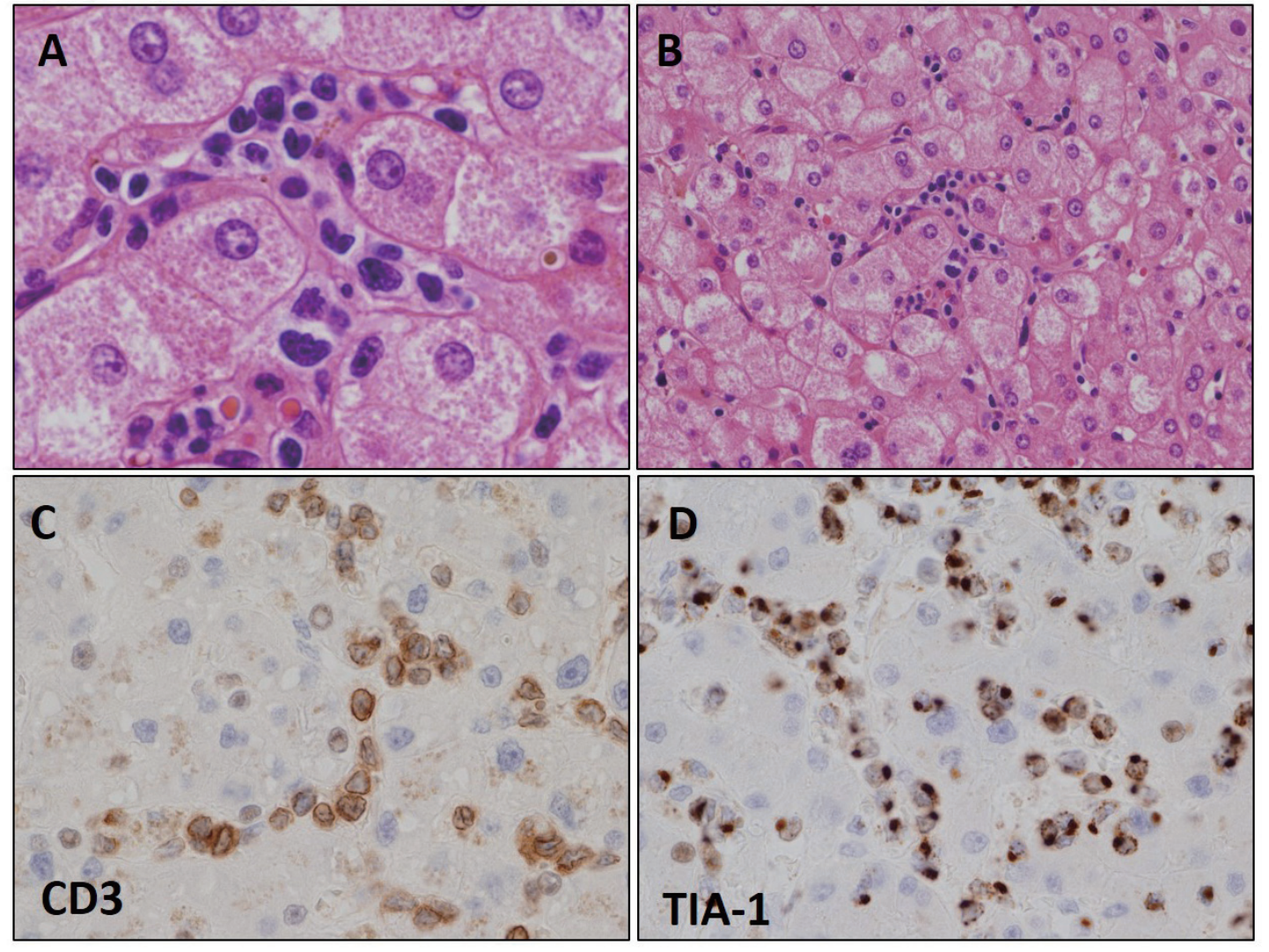

Fig. 3. Histological and immunohistochemical features of hepatosplenic T-cell lymphoma.

The tumor cells were small to medium in size with pale cytoplasm $(\boldsymbol{A}$ : HE x 400$)$. The liver exhibited predominant sinusoidal infiltration (B: HE x 200). Immunohistochemically, most cases were positive for CD3 ( $\boldsymbol{C}$ : CD3 x 400) and TIA-1 (D: TIA-1 x 400). The photographs in Figure 3 were kindly provided by Dr. Ohshima, Kurume University.

Overall, the clinicopathological findings of HSTL in IBD patients were not notably different from those of HSTL in immunocompetent patients.

\section{Therapy and prognosis for HSTL in IBD patients}

Information on the treatment and outcome was available for 47 of the 52 previously reported cases. Most patients received chemotherapy such as CHOP (combination of cyclophosphamide, vincristine, Adriamycin, and prednisolone), hyper CVAD (combination of cyclophosphamide, vincristine, doxorubicin, and dexamethasone), IVAC (combination of ifosfamide, etoposide, and cytarabine), and ICE (combination of ifosfamide, carboplatin, and etoposide). The outcomes of the patients were markedly poor. Only eight of 47 patients were alive at the time of the last followup. Information on transplantation was available for 27 cases. Eight patients received allogenic stem cell transplantation (SCT), five received autologous SCT, three received allogenic bone marrow transplantation, and 11 did not undergo transplantation. Among the 14 patients who received transplantation, seven were alive at the time of the last follow up. On the other hand, two of the 11 patients who did not receive transplantation were alive. In some cases, immunosuppressive drugs were withdrawn as the initial management after diagnosis. However, the tumor did not exhibit SR and the patients received chemotherapy. ${ }^{40,42}$

As mentioned above, HSTL in IBD patients was characterized by a markedly poor prognosis. Although the number of reported cases is small, SR after cessation of immunosuppressive agents was not expected. Therefore, the patients should receive chemotherapy as first-line treatment. Transplantation should be also considered because it may improve the prognosis. Indeed, although the enrolled patients were not limited to those with IBD, recent reports revealed that long-term survival may be expected in HSTL patients who receive SCT, particularly allogenic SCT. ${ }^{48,51,52}$ The graft-versus-lymphoma effect conferred by allogenic SCT was considered beneficial for the patients. In addition, Yabe et al. reported that hyper CVAD, an intensive chemotherapy, may result in better survival than a non-hyper CVAD regimen in HSTL patients. ${ }^{19}$

\section{CONCLUSION}

In this review, we mainly focused on summarizing the clinicopathological characteristics of MTX T-LPDs, MTX NK/T-LPDs, and HSTL in patients with IBD. The MTX T-LPD cases mainly consisted of three types: AITL, PTCLNOS, and $\mathrm{CD}^{+}{ }^{+}$T-LPD. The $\mathrm{EBV}^{+}$rate of MTX T-LPDs was $12 \%$, which is significantly lower than that for MTX 
B-LPDs. Notably, all of the $\mathrm{EBV}^{+}$cases were $\mathrm{CD} 8^{+} \mathrm{T}$-LPDs. SR may be expected in the majority of MTX T-LPD and NK/ T-LPD cases. In particular, all of the CD8 ${ }^{+}$T-LPD patients achieved CR after MTX cessation and none of the patients experienced relapse. As for the MTX B-LPD and HL type, withdrawal of MTX should be the initial management for MTX T-LPD and NK/T-LPD patients after diagnosis. In patients with IBD, the use of thiopurines and/or TNF antagonist may increase the risk for developing HSTL. The clinical and pathological features of HSTL in IBD overlap with those of HSTL in immunocompetent patients.

Due to the rarity of T-cell and NK-cell OIIA-LPDs, the number of previously reported cases is limited; therefore, more cases are needed to further clarify their features. Moreover, the molecular features and mechanism of pathogenesis remained to be elucidated.

\section{CONFLICT OF INTEREST}

The authors do not have any conflicts of interest to declare.

\section{REFERENCES}

1 Swerdlow SH, Campo E, Harris NL, et al. World Health Organization classification of hematopoietic and lymphoid tissue. Revised 4th ed, Lyon, International Agency for Research on Cancer. 2017; pp. 453-464.

2 Hoshida Y, Xu JX, Fujita S, et al. Lymphoproliferative disorders in rheumatoid arthritis: clinicopathological analysis of 76 cases in relation to methotrexate medication. J Rheumatol. 2007; $34: 322-331$.

3 Ichikawa A, Arakawa F, Kiyasu J, et al. Methotrexate/iatrogenic lymphoproliferative disorders in rheumatoid arthritis: histology, Epstein-Barr virus, and clonality are important predictors of disease progression and regression. Eur J Haematol. 2013; 91 : 20-28.

4 Mariette X, Cazals-Hatem D, Warszawki J, et al.; Investigators of the Club Rhumatismes et Inflammation. Lymphomas in rheumatoid arthritis patients treated with methotrexate: a 3-year prospective study in France. Blood. 2002; 99 : 3909-3915.

5 Yamakawa N, Fujimoto M, Kawabata D, et al. A clinical, pathological, and genetic characterization of methotrexate-associated lymphoproliferative disorders. J Rheumatol. 2014; 41 : 293-299.

6 Satou A, Tabata T, Miyoshi H, et al. Methotrexate-associated lymphoproliferative disorders of T-cell phenotype: clinicopathological analysis of 28 cases. Mod Pathol. 2019. [Epub ahead of print]

7 Kondo S, Tanimoto K, Yamada K, et al. Mature T/NK-cell lymphoproliferative disease and Epstein-Barr virus infection are more frequent in patients with rheumatoid arthritis treated with methotrexate. Virchows Arch. 2013; 462 : 399-407.

8 Hatanaka K, Nakamura N, Kojima M, et al. Methotrexateassociated lymphoproliferative disorders mimicking angioimmunoblastic T-cell lymphoma. Pathol Res Pract. 2010; 206 : 9-13.
9 Ishibuchi H, Motegi S, Yamanaka M, Amano H, Ishikawa O. Methotrexate-associated lymphoproliferative disorder: Sequential development of angioimmunoblastic T-cell lymphoma-like lymphoproliferation in the lymph nodes and diffuse large B-cell lymphoma in the skin in the same patient. Eur J Dermatol. 2015; $25: 361-362$.

10 Claudino WM, Gibson B, Tse W, Krem M, Grewal J. Methotrexateassociated primary cutaneous CD30-positive cutaneous T-cell lymphoproliferative disorder: a case illustration and a brief review. Am J Blood Res. 2016; 6 : 1-5.

11 Saleh JZ, Lee LH, Schieke SM, Hosking PR, Hwang ST. Methotrexate-induced $\mathrm{CD} 30^{+} \mathrm{T}$-cell lymphoproliferative disorder of the oral cavity. JAAD Case Rep. 2016; 2 : 354-356.

12 Koji H, Yazawa T, Nakabayashi K, et al. CD8-positive T-cell lymphoproliferative disorder associated with Epstein-Barr virus-infected B-cells in a rheumatoid arthritis patient under methotrexate treatment. Mod Rheumatol. 2016; 26 : 271-275.

13 Hatachi S, Kunitomi A, Aozasa K, Yagita M. CD8 ${ }^{+}$T-cell lymphoproliferative disorder associated with Epstein-Barr virus in a patient with rheumatoid arthritis during methotrexate therapy. Mod Rheumatol. 2010; $20: 500-505$.

14 Takajo I, Umekita K, Ikei Y, Oshima K, Okayama A. Adult T-cell Leukemia/Lymphoma as a Methotrexate-associated Lymphoproliferative Disorder in a Patient with Rheumatoid Arthritis. Intern Med. 2018; 57 : 2071-2075.

15 Nemoto Y, Taniguchi A, Kamioka M, et al. Epstein-Barr virusinfected subcutaneous panniculitis-like T-cell lymphoma associated with methotrexate treatment. Int J Hematol. 2010; 92 : 364-368.

16 Terroso G, Aleixo J, Bernardes M, et al. Nasal type extranodal NK/T cell lymphoma diagnosed in a patient with rheumatoid arthritis under methotrexate. Acta Reumatol Port. 2014; 39 : 77-81.

17 Tajima S, Takanashi Y, Koda K, Fukayama M. Methotrexateassociated lymphoproliferative disorder presenting as extranodal NK/T-cell lymphoma arising in the lungs. Pathol Int. 2015; $65: 661-665$.

18 Gratzinger D, de Jong D, Jaffe ES, et al. T- and NK-Cell Lymphomas and Systemic Lymphoproliferative Disorders and the Immunodeficiency Setting: 2015 SH/EAHP Workshop Report-Part 4. Am J Clin Pathol. 2017; 147 : 188-203.

19 Yabe M, Miranda RN, Medeiros LJ. Hepatosplenic T-cell Lymphoma: a review of clinicopathologic features, pathogenesis, and prognostic factors. Hum Pathol. 2018; 74 : 5-16.

20 Yabe M, Medeiros LJ, Tang G, et al. Prognostic Factors of Hepatosplenic T-cell Lymphoma: Clinicopathologic Study of 28 Cases. Am J Surg Pathol. 2016; 40 : 676-688.

21 Thai A, Prindiville T. Hepatosplenic T-cell lymphoma and inflammatory bowel disease. J Crohns Colitis. 2010; 4 : 511-522.

22 Rath T, Rubbert A. Drug combinations with methotrexate to treat rheumatoid arthritis. Clin Exp Rheumatol. 2010; 28(suppl 61) : S52-S57.

23 Thomas E, Brewster DH, Black RJ, Macfarlane GJ. Risk of malignancy among patients with rheumatic conditions. Int $\mathrm{J}$ Cancer. 2000; $88:$ 497-502.

24 Mellemkjær L, Linet MS, Gridley G, et al. Rheumatoid arthritis and cancer risk. Eur J Cancer. 1996; 32 : 1753-1757. 
25 Gridley G, McLaughlin JK, Ekbom A, et al. Incidence of cancer among patients with rheumatoid arthritis. J Natl Cancer Inst. 1993; $85: 307-311$.

26 Gion Y, Iwaki N, Takata K, et al. Clinicopathological analysis of methotrexate-associated lymphoproliferative disorders: Comparison of diffuse large B-cell lymphoma and classical Hodgkin lymphoma types. Cancer Sci. 2017; 108 : 1271-1280.

27 Kamel OW, van de Rijn M, Weiss LM, et al. Brief report: reversible lymphomas associated with Epstein-Barr virus occurring during methotrexate therapy for rheumatoid arthritis and dermatomyositis. N Engl J Med. 1993; 328 : 1317-1321.

28 Feng WH, Cohen JI, Fischer S, et al. Reactivation of latent Epstein-Barr virus by methotrexate: a potential contributor to methotrexate-associated lymphomas. J Natl Cancer Inst. 2004; $96: 1691-1702$.

29 Landais E, Saulquin X, Houssaint E. The human T cell immune response to Epstein-Barr virus. Int J Dev Biol. 2005; 49 : 285-292.

30 Masucci MG, Ernberg I. Epstein-Barr virus: adaptation to a life within the immune system. Trends Microbiol. 1994; 2 : 125-130

31 Sandhu A, Ahmad S, Kaur P, et al. Methotrexate preferentially affects Tc1 and Tc17 subset of CD8 T lymphocytes. Clin Rheumatol. 2019; 38 : 37-44.

32 Farcet JP, Gaulard P, Marolleau JP, et al. Hepatosplenic T-cell lymphoma: sinusal/sinusoidal localization of malignant cells expressing the T-cell receptor gamma delta. Blood. 1990; 75 : 2213-2219.

33 Macon WR, Levy NB, Kurtin PJ, et al. Hepatosplenic alphabeta T-cell lymphomas: a report of 14 cases and comparison with hepatosplenic gammadelta T-cell lymphomas. Am J Surg Pathol. 2001; 25 : 285-296.

34 Herrinton LJ, Liu L, Weng X, et al. Role of thiopurine and antiTNF therapy in lymphoma in inflammatory bowel disease. Am J Gastroenterol. 2011; $106: 2146-2153$

35 Mackey AC, Green L, Liang LC, Dinndorf P, Avigan M. Hepatosplenic $\mathrm{T}$ cell lymphoma associated with infliximab use in young patients treated for inflammatory bowel disease. J Pediatr Gastroenterol Nutr. 2007; 44 : 265-267.

36 Mackey AC, Green L, Leptak C, Avigan M. Hepatosplenic T cell lymphoma associated with infliximab use in young patients treated for inflammatory bowel disease: update. J Pediatr Gastroenterol Nutr. 2009; 48 : 386-388.

37 Deepak P, Sifuentes H, Sherid M, et al. T-cell non-Hodgkin's lymphomas reported to the FDA AERS with tumor necrosis factor-alpha (TNF- $\alpha$ ) inhibitors: results of the REFURBISH study. Am J Gastroenterol. 2013; 108 : 99-105.

38 Kotlyar DS, Osterman MT, Diamond RH, et al. A systematic review of factors that contribute to hepatosplenic T-cell lymphoma in patients with inflammatory bowel disease. Clin Gastroenterol Hepatol. 2011; 9 : 36-41.e1.

39 Navarro JT, Ribera JM, Mate JL, et al. Hepatosplenic T-gammadelta lymphoma in a patient with Crohn's disease treated with azathioprine. Leuk Lymphoma. 2003; 44 : 531-533.
40 Mittal S, Milner BJ, Johnston PW, Culligan DJ. A case of hepatosplenic gamma-delta T-cell lymphoma with a transient response to Fludarabine and Alemtuzumab. Eur J Haematol. 2006; $76: 531-534$.

41 Zeidan A, Sham R, Shapiro J, Baratta A, Kouides P. Hepatosplenic T-cell lymphoma in a patient with Crohn's disease who received infliximab therapy. Leuk Lymphoma. 2007; 48 : 1410-1413.

42 Humphreys MR, Cino M, Quirt I, Barth D, Kukreti V. Longterm survival in two patients with hepatosplenic T cell lymphoma treated with interferon- alpha. Leuk Lymphoma. 2008; 49 : 1420-1423.

43 Moran G, Dillon J, Green J. Crohn's disease, hepatosplenic T-cell lymphoma and no biological therapy: are we barking up the wrong tree? Inflamm Bowel Dis. 2009; 15 : 1281-1282.

44 Ochenrider MG, Patterson DJ, Aboulafia DM. Hepatosplenic T-cell lymphoma in a young man with Crohn's disease: case report and literature review. Clin Lymphoma Myeloma Leuk. 2010; $10: 144-148$.

45 Côté-Daigneault J, Bernard EJ. Hepatosplenic lymphoma presenting initially as hemophagocytic syndrome in a 21-year-old man with Crohn's disease: a case report and literature review. Can J Gastroenterol. 2011; 25 : 417-418.

46 Bašić Kinda S, Duraković N, Dotlić S, et al. Hepatosplenic $\alpha \beta$ T-cell lymphoma arising after long-term azathioprine therapy successfully treated with allogeneic bone marrow transplant. Leuk Lymphoma. 2013; 54 : 1334-1335.

47 Subramaniam K, Yeung D, Grimpen F, et al. Hepatosplenic T-cell lymphoma, immunosuppressive agents and biologicals: what are the risks? Intern Med J. 2014; 44 : 287-290.

48 Voss MH, Lunning MA, Maragulia JC, et al. Intensive induction chemotherapy followed by early high-dose therapy and hematopoietic stem cell transplantation results in improved outcome for patients with hepatosplenic T-cell lymphoma: a single institution experience. Clin Lymphoma Myeloma Leuk. 2013; $13: 8-14$.

49 Parakkal D, Sifuentes H, Semer R, Ehrenpreis ED. Hepatosplenic T-cell lymphoma in patients receiving TNF- $\alpha$ inhibitor therapy: expanding the groups at risk. Eur J Gastroenterol Hepatol. 2011; $23: 1150-1156$.

50 Biancone L, Onali S, Petruzziello C, Calabrese E, Pallone F. Cancer and immunomodulators in inflammatory bowel diseases. Inflamm Bowel Dis. 2015; 21 : 674-698.

51 Tanase A, Schmitz N, Stein H, et al.; Lymphoma Working Party of the EBMT. Allogeneic and autologous stem cell transplantation for hepatosplenic T-cell lymphoma: a retrospective study of the EBMT Lymphoma Working Party. Leukemia. 2015; 29 : 686-688.

52 Rashidi A, Cashen AF. Outcomes of allogeneic stem cell transplantation in hepatosplenic T-cell lymphoma. Blood Cancer J. $2015 ; 5$ : e318. 